\title{
Per2 participates in AKT-mediated drug resistance in A549/DDP lung adenocarcinoma cells
}

\author{
BO CHEN, YAOXI TAN, YAN LIANG, YAN LI, LEI CHEN, SHUANGSHUANG WU, \\ WEI XU, YAN WANG, WEIHONG ZHAO and JIANQING WU
}

Department of Geriatrics, The First Affiliated Hospital of Nanjing Medical University, Nanjing, Jiangsu 210029, P.R. China

Received May 26, 2015; Accepted April 15, 2016

DOI: $10.3892 / 01.2016 .5430$

\begin{abstract}
Period2 (Per2) is a key mammalian circadian clock protein, and additionally has a tumor suppressive function. The present study aimed to investigate its role in drug resistance in A549/cisplatin (DDP) lung adenocarcinoma cells. Per2 knockdown and overexpression in A549/DDP cells were used to compare cell proliferation (by MTT assay), apoptosis (active-caspase 3 western blot) and clone forming assay. The activation of AKT/mechanistic target of rapamycin (mTOR) was investigated by a western blot assay. The Per2 expression level was decreased in A549/DDP cells compared with A549 cells. Per2 knockdown by short hairpin RNA protects A549/DDP cells from apoptosis, and promotes proliferation and migration. Per2 knockdown results in increased activation of the phosphoinositide 3-kinase (PI3K)/AKT/mTOR signaling pathway. Overexpression of Per2 in A549/DDP cells may reduce the activity of the $\mathrm{PI} 3 \mathrm{~K} / \mathrm{AKT} / \mathrm{mTOR}$ signaling pathway, and promote apoptosis of A549 cells. The results of the present study suggest that Per2 participates in AKT-mediated drug resistance in A549/DDP lung adenocarcinoma cells.
\end{abstract}

\section{Introduction}

Platinum-based drugs, one of the most effective types of anti-cancer treatment, are widely used in the treatment of almost all types of solid tumors (1). Cisplatin (DDP) treatment frequently leads to the acquisition of chemoresistance (1). Numerous mechanisms underlying this chemoresistance have been identified; studies have documented that DDP may trigger the activation of Jun-N-terminal kinase and p38

Correspondence to: Professor Jianqing Wu, Department of Geriatrics, The First Affiliated Hospital of Nanjing Medical University, 300 Guangzhou Road, Nanjing, Jiangsu 210029, P.R. China

E-mail: jwuny@njmu.ed.cn

Key words: period2, lung cancer, chemoresistance, phosphoinositide 3-kinase/AKT/mechanistic target of rapamycin, apoptosis mitogen-activated protein kinase cascades in tumor cells or transformed cell lines (2-4). Protein kinase B (PKB/AKT) is a cytoplasmic serine/threonine kinase that positively regulates metabolism, cell cycle progression and cell survival $(5,6)$. It has been reported that DDP activates PKB/AKT in several cancer cell lines (4). Inhibition of phosphoinositide 3-kinase (PI3K) activity decreases the survival of cells exposed to DDP, suggesting that cisplatin-induced PKB/Akt activation may lead to cisplatin resistance (4). Activation of ERK1/2 and AKT has also been reported to be associated with cisplatin resistance in human lung cancer cells (7). Improvement of our understanding of the cellular responses to cisplatin remains important to optimize its use in the clinic.

Period2 (Per2) is a circadian gene $(8,9)$. Mounting evidence suggests that deregulation of the circadian clock has a significant role in the development of mammalian cancer (8-11). Studies have revealed that the expression of Per2 in non-small cell lung cancer (NSCLC) is decreased $(8,9)$. Negative expression of Per2 may contribute to development and invasion in NSCLC (8). To the best of our knowledge, whether Per2 deregulation is associated with cisplatin resistance in lung cancer cells remains to be elucidated.

To investigate the role of Per2 in cisplatin resistance in lung cancer cells, the present study used A549/DDP cells as a working system. Initially, the expression of Per2 in A549 and A549/DDP cells was determined. Subsequently, the function of Per2 in A549/DDP cells was investigated by gene silencing approaches. In addition, western blot analyses were performed to identify signaling pathways that mediate the effects of Per2. Overall, the present study shows that Per2 is downregulated in A549/DDP cells, and it regulates the biological function of A549/DDP cells via the AKT/mechanistic target of rapamycin (mTOR) signaling pathway.

\section{Materials and methods}

Antibodies. Anti-pS473AKT (cat. no. 4060; 1:1,000), anti-pS2448mTOR (cat. no. 2971; 1:2,000), anti-mTOR (cat. no. $2972 ; 1: 2,000)$ and anti- $\alpha$-actin (cat. no. 4970; $1: 5,000$ ) antibodies were purchased from Cell Signaling Technology, Inc. (Danvers, MA, USA) and the anti-AKT antibody (cat. no. sc-135829; 1:1,000) was purchased from Santa Cruz Biotechnology, Inc. (Dallas, TX, USA). Rabbit anti-active caspase-3 (cat. no. ab13585; 1:3,000) and anti-Per2 (cat. 
no. ab179813; 1:1,000) antibodies were purchased from Abcam (Cambridge, UK). The horseradish peroxidase (HRP)-conjugated secondary antibody (cat. no. ab6721; 1:5,000) was also purchased from Abcam.

Cell lines. The human lung adenocarcinoma cell line A549 (Type Culture Collection of the Chinese Academy of Sciences, Shanghai, China). was cultured in Dulbecco's modified Eagle's medium (HyClone; GE Healthcare Life Sciences, Logan, UT, USA). A549/DDP cells were cultured in RPMI-1640 medium (HyClone; GE Healthcare Life Sciences). The medium was supplemented with $10 \%$ fetal bovine serum (HyClone; GE Healthcare Life Sciences), and cells were maintained at $37^{\circ} \mathrm{C}$ in a humidified atmosphere of $5 \% \mathrm{CO}_{2}$. The cells were subcultured when they reached $\sim 90 \%$ confluence using a $0.25 \%$ trypsin solution.

Per2 overexpression. Human Per2 was amplified according to the published sequence (accession no. AB012614; https://www.ncbi.nlm.nih.gov/nuccore/AB012614) using specific primers, and cloned into pcDNA 3.1 (Invitrogen; Thermo Fisher Scientific, Inc.). Following confirmation by sequencing, the construct was transfected into A549/DDP cells using Lipofectamine ${ }^{\mathrm{TM}} 2000$ reagent (Invitrogen; Thermo Fisher Scientific, Inc.) according to the manufacturer's instructions.

Knockdown of Per2 by short hairpin (sh)RNA. The pSuper vectors (OligoEngine, Seattle, WA, USA) were used to transcribe functional shRNA. In the vectors, oligonucleotides targeting Per2 were inserted downstream of the $\mathrm{H} 1$ promoter, with their veracity confirmed by double digestion and sequencing. The target sequences were as follows: shRNA1, GCGTTACCTCTGAGCACATTG; shRNA2, GCATGGAGG AGAAATCTTTCT; shRNA3: GGAGTTAGAGATGGTGGA AGA; shRNA4, GCTGCCTTCCCGAAATTTAGA. Transient transfection of constructs in A549 cells was performed using Lipofectamine $^{\mathrm{TM}} 2000$ reagent (Invitrogen; Thermo Fisher Scientific, Inc.) according to the manufacturer's protocol.

Reverse transcription-quantitative polymerase chain reaction (RT-qPCR). Transcripts were quantified by RT-qPCR. Briefly, total RNA was extracted from the cells and reverse transcribed into cDNA using the First Strand cDNA Synthesis kit (CWBIO, Beijing, China). Subsequently, qPCR was performed using the UltraSYBR Mixture (CWBIO) on a thermocycler with the following cycling conditions: 36 cycles of denaturation at $95^{\circ} \mathrm{C}$ for $50 \mathrm{sec}$, annealing at a temperature dependent on the melting temperature of each primer set for $30 \mathrm{sec}$ and an extension step at $72^{\circ} \mathrm{C}$ for $30 \mathrm{sec}$. Primer sequences used were as follows: Per2 forward, 5'-GAGACCCAGTCCTGTTTGGT-3' and reverse, 5'-ATACAGATGCAGTCGCAAGC-3'. Primers specific for GAPDH were used as a control (forward, 5'-CAT CTTCTTTTGCGTCGCCA-3' and reverse, 5'-TTAAAAGCA GCCCTGGTGACC-3'). The relative expression levels of Per2 mRNA were calculated using the $2^{-\Delta \Delta \mathrm{Cq}}$ method and normalized to GAPDH (10).

Protein extraction and western blotting. Whole-cell lysate was extracted with radioimmunoprecipitation assay buffer in
A

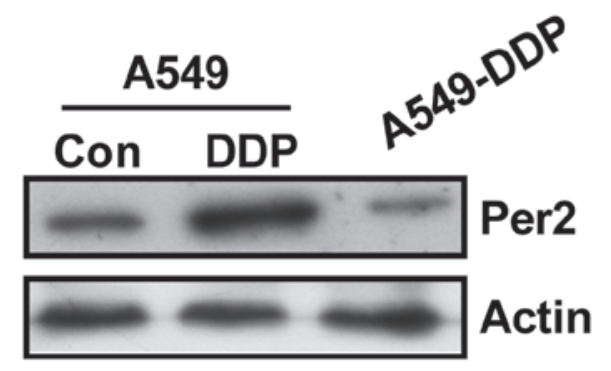

B

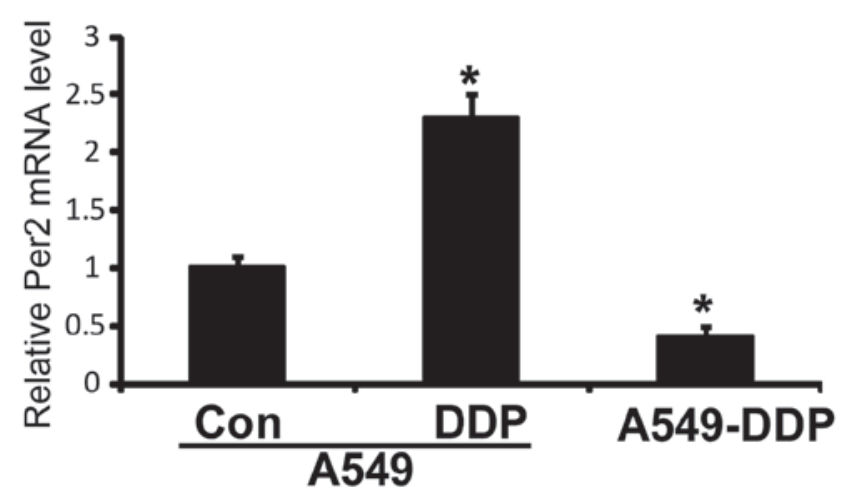

Figure 1. Expression of Per2 in A549/DDP cells. (A) Western blot analysis of Per2 was performed. $\beta$-actin was used as an internal control. (B) Quantitative polymerase chain reaction was performed to demonstrate the relative mRNA level of Per2. Data are presented as the mean \pm standard deviation; $n=3$ ) ${ }^{*} \mathrm{P}<0.05$ vs. control cells. Per2, period2; Con, control.

the presence of complete protease inhibitor cocktail (Roche Diagnostics, Indianapolis, IN, USA). Protein quantification was performed using the performed using Pierce BCA Protein Assay kit (Thermo Fisher Scientific, Inc.). Protein levels were measured by western blotting. Briefly, equal amounts of protein $(25 \mu \mathrm{g})$ were separated by $10 \%$ SDS-PAGE, and transferred to polyvinylidene membranes (Bio-Rad Laboratories, Inc., Hercules, CA, USA). The membranes were incubated in blocking buffer $(0.2 \mathrm{mM}$ Tris, $137 \mathrm{mM} \mathrm{NaCl}, 5 \%$ no-fat milk and $0.1 \%$ Tween-20) for $1 \mathrm{~h}$ at room temperature and then probed at $4^{\circ} \mathrm{C}$ overnight with the primary antibodies against pS473AKT, pS2448mTOR, mTOR, AKT, caspase-3, Per2 and $\alpha$-actin. The membranes were rinsed with washing buffer (0.1\% Tween 20,0.2 $\mathrm{mM}$ Tris and $137 \mathrm{mM} \mathrm{NaCl}$ ) and incubated with HRP-conjugated secondary antibody $(1: 5,000)$ for $1 \mathrm{~h}$ at room temperature, followed by chemiluminescence detection (Luminata Western HRP Substrate; EMD Millipore, Billerica, MA, USA). The defined sections of the film were scanned for density measurement and analyzed using ImageJ v2 1.4.7 software (National Institutes of Health, Bethesda, MD, USA).

Wound healing assay. A total of $3 \times 10^{5}$ A549/DDP cells were plated in a 6 -well plate. When cell confluence was $<80 \%$ at $48 \mathrm{~h}$ subsequent to transfection, wounds were created in confluent cells using a 200-ml pipette tip. The cells were subsequently rinsed with medium to remove free-floating cells and debris. Medium was subsequently added, and culture plates were incubated at $37^{\circ} \mathrm{C}$ for $48 \mathrm{~h}$. Wound healing within the scrape line was observed at $24 \mathrm{~h}$, and representative scrape lines for each group were photographed. Duplicate wells for each condition were examined for each experiment, and each 
A

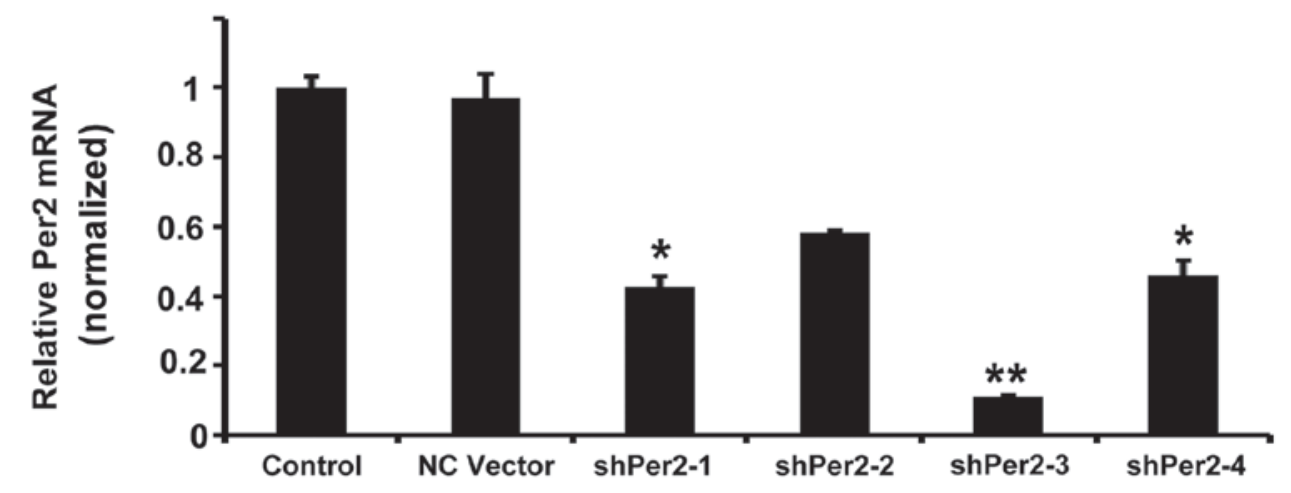

B

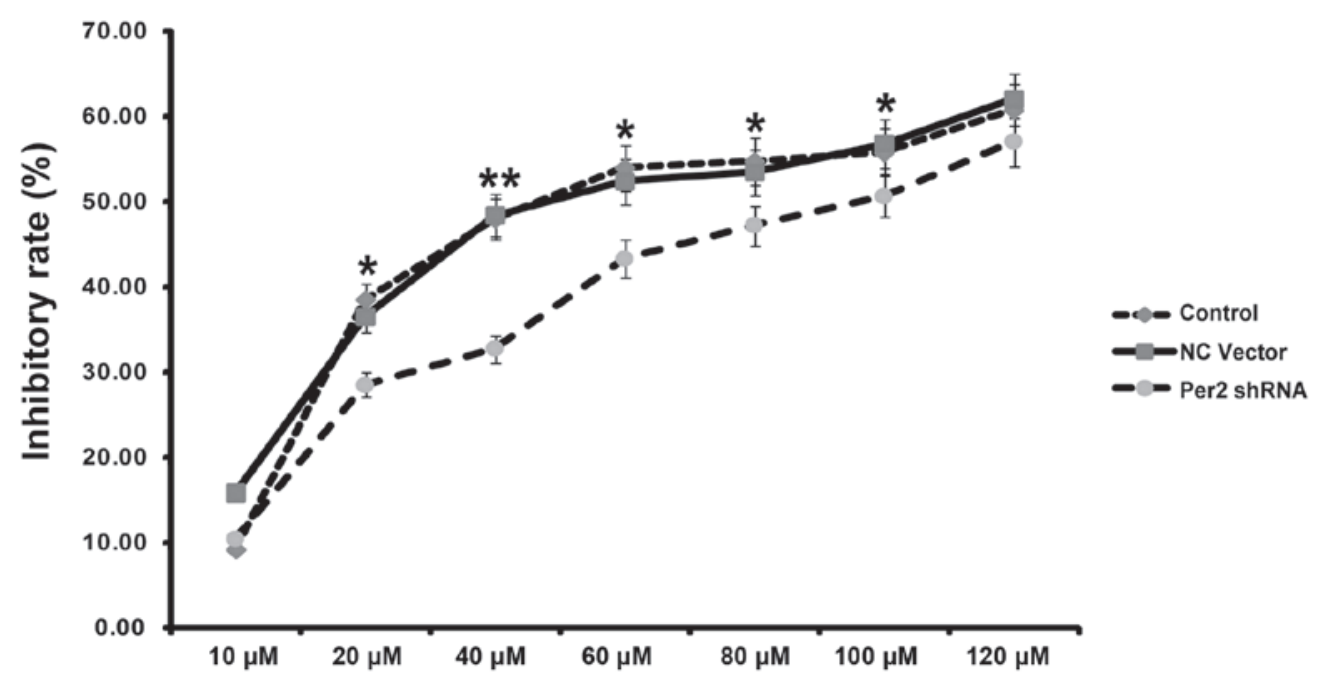

Figure 2. Effect of Per2 knockdown by shRNA on A549/DDP cell proliferation. (A) Quantitative polymerase chain reaction analysis of Per2 was performed. (B) Effects of proliferation on A549/DDP cells treated with DDP by MTT assay. Data are presented as the mean \pm standard deviation; $\mathrm{n}=3$. " $\mathrm{P}<0.05$, ${ }^{* *} \mathrm{P}<0.01$ vs. the NC vector. Per2, period2; sh, short hairpin; NC, negative control.

experiment was repeated 3 times. The sizes of the wounds were measured using ImageJ v2.1.4.7 software.

MTT and clonogenic assay. Cell proliferation was assessed using conversion of 3-[4,5-dimethylthiazol-2-yl]-2,5-diphenyltetrazolium bromide (Sigma-Aldrich; EMD Millipore) to formazan product at 24-h intervals. For clonogenic assay, A549/DDP cells transfected with shPer2 vector were plated at equal density $(1,000 /$ well $)$ in $35-\mathrm{mm}$ culture dishes for 14 days. The cells were rinsed with PBS prior to staining with $0.25 \%$ crystal violet $/ 20 \%$ ethanol for $5 \mathrm{~min}$ and the number of colonies was counted manually.

Statistical analysis. Experimental data are presented as the mean \pm standard error. All statistical analysis was performed with use of SPSS version 13.5 (SPPS, Inc., Chicago, IL, USA). Student's t-test and one-way analysis of variance, followed by Tukey's post hoc analysis, was applied. $\mathrm{P}<0.05$ was considered to indicate a statistically significant difference.

\section{Results}

Expression of Per2 is downregulated in A549/DDP cells compared with A549 cells. Western blotting was performed to determine the expression of Per2 in A549 and A549/DDP cells. As shown in Fig. 1A, DDP treatment caused an increase in Per2 protein expression, while the A549/DDP cells demonstrated marked downregulation of Per2 compared with A549 cells. To determine whether downregulation of Per2 expression was a result of reduced transcription, Per2 mRNA was also analyzed by RT-qPCR. Downregulated levels of Per2 mRNA were also detected (Fig. 1B). These results indicated that expression of Per2 was downregulated in A549/DDP cells compared with A549 cells.

Per2 knockdown in A549/DDP cells by shRNA protects from apoptosis, but promotes proliferation and migration. To investigate the potential role of Per2 in lung cancer, the present study examined various biological functions of A549/DDP cells upon Per2 knockdown by shRNA. Effective inhibition of Per2 was demonstrated by semiquantitative RT-PCR (Fig. 2A). RT-qPCR indicated that the shRNA1 and shRNA3 reduced Per 2 mRNA levels to 42 and $11 \%$, respectively, compared with the controls (mean level of the nonspecific RNA control). Subsequently, the present study used shRNA3 for additional examination. In order to observe the impact of Per2 on the proliferation of A549/DDP cells, the inhibition rates of A549/DDP cells treated with various 
A

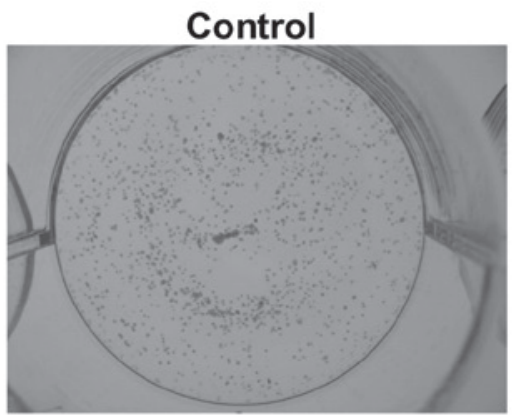

B

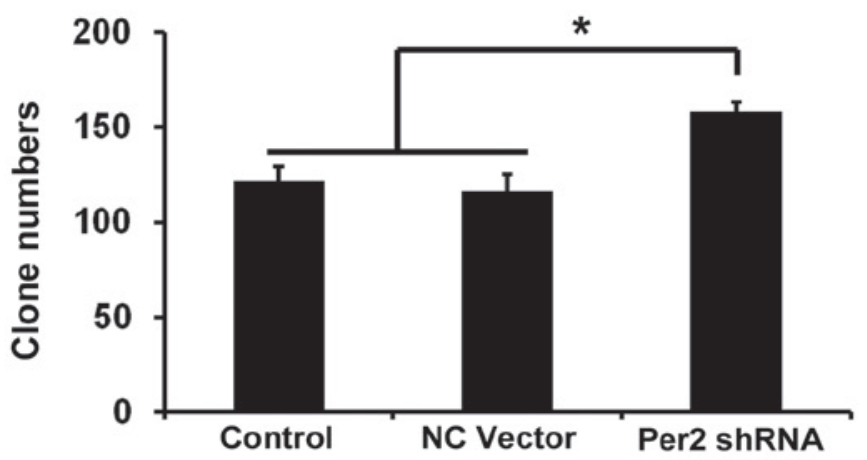

NC Vector

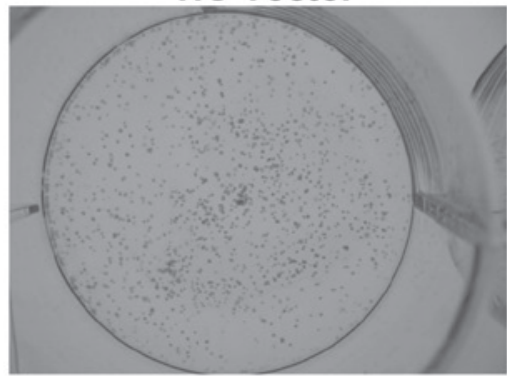

$\mathbf{C}$
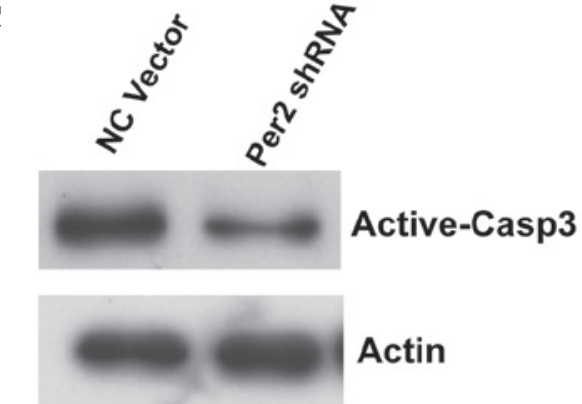

Actin

Figure 3. Effect of Per2 knockdown by shRNA on clonogenic ability and apoptosis of A549/DDP cells. (A) Photographs of colonies from control and NC vector or Per2 shRNA are shown (magnification, $\mathrm{x} 40$ ). (B) The colony numbers were counted. Data are presented as the mean \pm standard deviation; $\mathrm{n}=3$. ${ }^{*} \mathrm{P}<0.05$. (C) Western blot analysis of active-caspase 3 was performed. $\beta$-Actin was used as an internal control. Per2, period2; sh, short hairpin; NC, negative control; Casp, caspase.

A

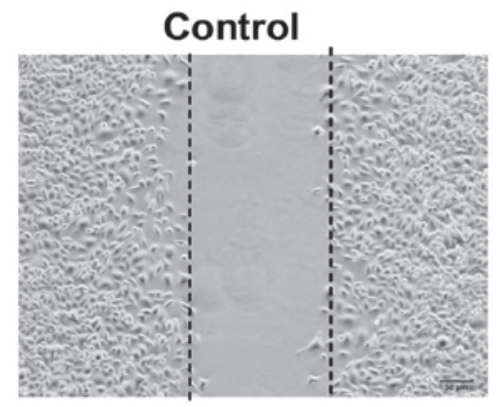

NC Vector

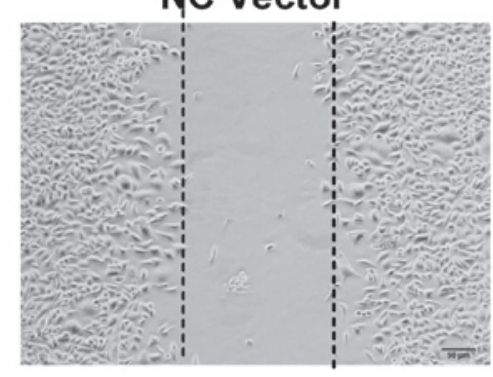

Per2 shRNA

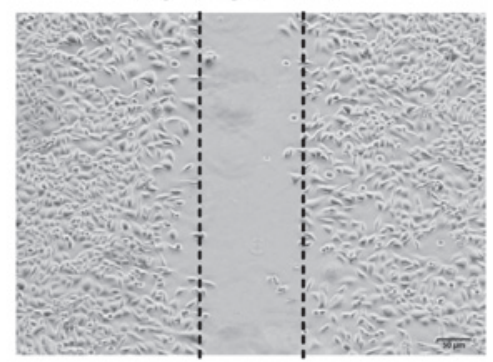

B

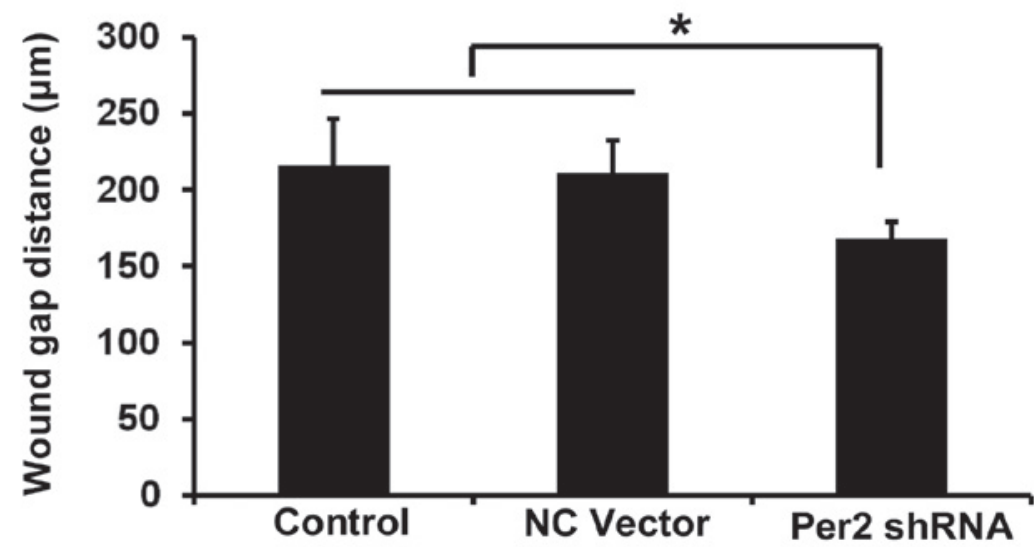

Figure 4. Effect of Per2 knockdown by shRNA on cell motility of A549/DDP cells. (A) Scratch wound closure assay was performed for motility of A549/DDP cells transfected with indicated constructs. Images were captured $48 \mathrm{~h}$ subsequent to the scratch (magnification, x100). (B) Quantitative assays were performed by calculating the wound gap distance. Data are presented as the mean \pm standard error from three independent experiments. ${ }^{*}<0.05$. Per2, period2; sh, short hairpin; $\mathrm{NC}$, negative control. 
$\mathbf{A}$

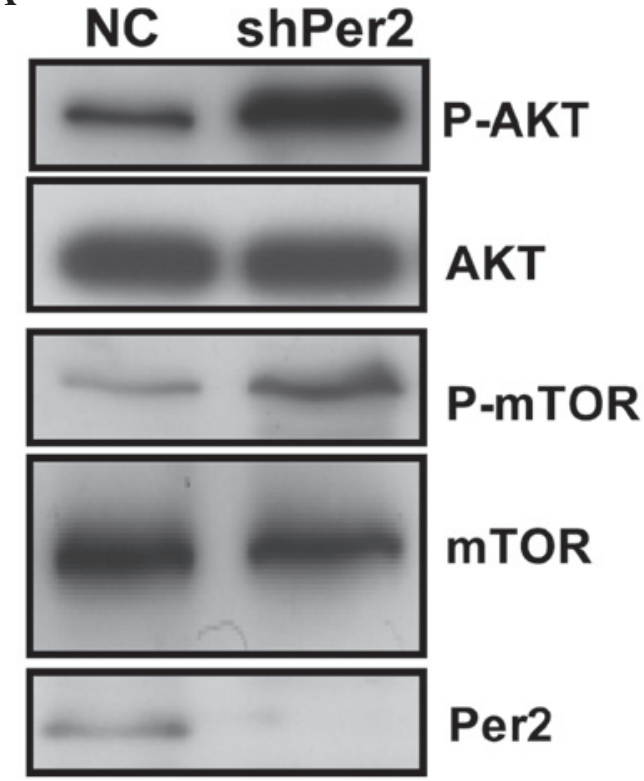

B

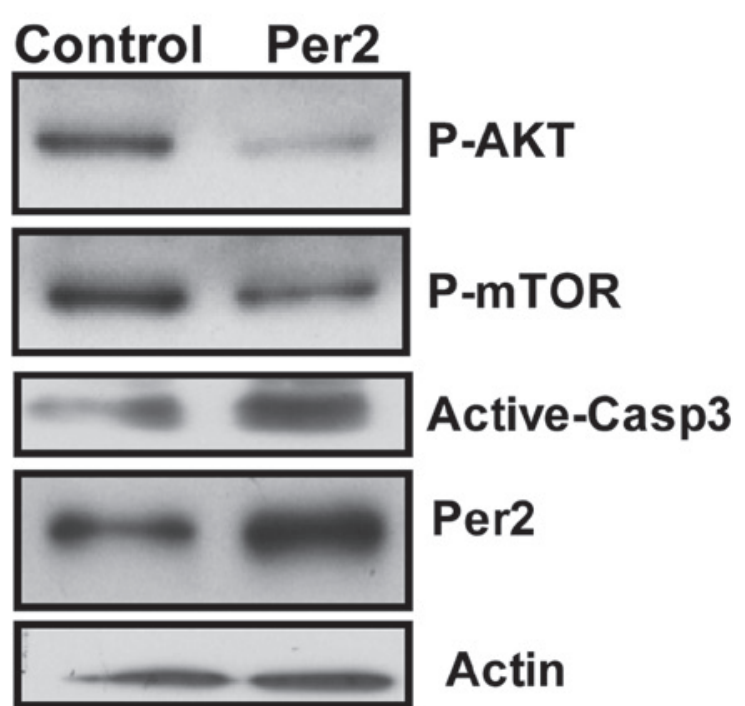

Figure 5. Effect of Per2 knockdown or overexpression on the phosphoinositide 3-kinase/AKT/mTOR and caspase-3 signaling pathways. The expression of various proteins in the cells following (A) Per2 knockdown and (B) Per 2 overexpression was examined by western blotting. $\beta$-Actin was used as an internal control. Per2, period2; mTOR, mechanistic target of rapamycin; NC, negative control; sh, short hairpin; p, phosphorylated; Casp, caspase.

concentrations of DDP at $24 \mathrm{~h}$ were calculated. As shown in Fig. 2B, the growth inhibition rate increased along with increasing concentration of DDP. Per2 knockdown decreases the inhibition rate of A549/DDP cells compared with controls, independent of the concentration of DDP (Fig. 2B). The $\mathrm{IC}_{50}$ values of DDP were $58.1 \mu \mathrm{mol} / 1$ for the nonspecific RNA control group, and $88.0 \mu \mathrm{mol} / 1$ for the sh-Per2 transfection group. In clonogenic assays, cells transfected with shPer2-RNA construct or NC vector construct, together with an untransfected control, were respectively plated out at low density. Following 2 weeks of culture, the number of colonies that attached to the substrate reflected the survival efficiency at low cell density. The shPer2-RNA transfectants showed increased colony formation ability by $136 \%$ compared with the vector control (Fig. 3A and B). Subsequently, the present study detected the apoptosis associated protein active-caspase- 3 by western blotting. Per 2 shRNA decreased the expression of active-caspase-3 in A549/DDP cells induced by serum-starvation for $48 \mathrm{~h}$ (Fig. 3C). These results indicate that Per2 knockdown promotes cell proliferation and survival.

The migration ability of A549/DDP cells was investigated by wound healing assay. The wound healing assay results revealed that the distance of migration in A549/DDP cells was significantly increased following transfection with shPer2 (Fig. 4A and B).

Per2 knockdown increases the activity of the PI3K/AKT/mTOR signaling pathway, and overexpression of Per 2 may reduce the activity and promote apoptosis of A549 cells. The present study investigated whether PI3K/AKT/mTOR, which is important in regulating cell proliferation and apoptosis, is involved in Per2-mediated cell death in A549/DDP cells. Per2 knockdown by shRNA was observed to cause a significant increase in the phosphorylation (Ser473) of AKT protein and (S2448) mTOR in cells (Fig. 5A). However, shRNA transfection did not cause any change in the protein levels of total AKT. This transfection resulted in increased levels of the phosphorylated (activated) form of mTOR (Ser2448), a downstream target of PI3K/AKT, which may promote cell growth. The results of the present study revealed a potent effect of Per2 on AKT/mTOR signaling.

To additionally determine whether the expression level of Per2 is involved in AKT/mTOR signaling in A549/DDP cells, expression plasmids for Per2 were constructed. The constructs were transfected into A549 cells for $48 \mathrm{~h}$. Ectopic expression of Per2 protein resulted in a dramatic reduction in the active form of AKT and mTOR. Furthermore, ectopic expression of Per2 increased the quantity of active-caspase-3 protein upon serum starvation stimuli (Fig. 5B). These results confirm the effect of Per2 on AKT/mTOR signaling, and indicate Per2 is functional in apoptosis regulation of A549/DDP cells.

\section{Discussion}

Lung cancer remains the leading cause of death among malignant tumors worldwide. The incidence of NSCLC, the most common form of lung cancer, is increasing (12). Due to insensitivity to cytotoxic agents, identifying molecules that drive lung cancer growth, survival and metastasis is critical.

Deregulation of the circadian clock has been implicated in numerous types of cancer $(8-11,13)$. The circadian clock proteins Per1 and Per 2 function in a series of cellular processes that coordinate the circadian clock in the brain and peripheral tissues (13). Mice that are deficient in the Per2 gene are more cancer-prone and sensitive to $\gamma$-irradiation (13). Overexpression of Per2 induces apoptosis and alters the expression levels of apoptosis-associated genes in tumor cells (13). Furthermore, loss of Per2 under hypoxia upregulates organic cation transporter 1-mediated epithelial-to-mesenchymal (EMT) gene expression and enhances tumor malignancy in breast cancer (14). In addition, cells with downregulated Per2 expression have prolonged high levels of AKT T308 phosphorylation following growth factor stimulation or DNA damage (14). However, whether Per2 has a role in lung cancer cells remains to be elucidated. 
In the present study, it is reported that Per2 participates in AKT-mediated drug resistance in A549/DDP lung adenocarcinoma cells. It was observed that the Per2 expression level is decreased in A549/DDP cells compared with A549 cells. Per2 knockdown by shRNA protects A549/DDP cells from apoptosis, while promoting its proliferation and migration. Finally, it was observed that Per2 knockdown results in increased activation of the PI3K/AKT/mTOR signaling pathway. The results of the present study provide two insights into the mechanism of Per2-dependent signaling regulation in A549/DDP cells.

Initially, it was observed that the DDP-resistant A549 cells demonstrated altered Per2 expression levels. Per2 expression was demonstrated to be significantly reduced in breast cancer and breast cancer stem cells $(15,16)$. It has also been indicated that the expression of Per2 in NSCLC is decreased (8). The results of the present study demonstrated that Per2 expression was significantly reduced in A549/DDP cells compared with A549 cells.

Furthermore, it was observed that Per2 knockdown affected the biological function of A549/DDP cells, potentially via the PI3K-AKT-mTOR signaling pathway. Per2 is thought to be a tumor suppressor, and is involved in numerous cancer cell functions, including EMT, invasion and apoptosis $(8-10,13,15)$. The results of the present study demonstrated that Per2 knockdown by shRNA protects A549/DDP cells from apoptosis, and promotes proliferation and migration. Overexpression of Per2 was able to promote apoptosis of A549/DDP cells. Furthermore, it was observed that Per 2 knockdown by shRNA caused a significant increase in the phosphorylation of AKT protein and mTOR in cells. By contrast, overexpression of Per2 could reduce the activity of PI3K-AKT-mTOR signaling. The results of the present study suggest that the activity of the PI3K/AKT/mTOR signaling pathway may have roles in Per2 functioning in A549/DDP cells.

In conclusion, using A549/DDP cells as a working system, the present study investigated the role of Per2 in DDP resistance in lung cancer cells. The present study demonstrates that Per2 is downregulated in A549/DDP cells, and that it regulates the biological function of A549/DDP cells via the AKT signaling pathway.

\section{Acknowledgements}

The present study was funded by the Priority Academic Program Development of Jiangsu Higher Education Institutions (Jiangsu, China) and was supported by grants from the
National Natural Science Foundation of China (Beijing, China; grant nos., 81572259, 81272602 and 81302011). The abstract was presented at the 2015 Chinese Congress on Gerontology and Health Industry. September 11 - September 13, 2015, Suzhou, Jiangsu, China.

\section{References}

1. Kartalou M and Essigmann JM: Mechanisms of resistance to cisplatin. Mutat Res 478: 23-43, 2001.

2. Deschesnes RG, Huot J, Valerie K and Landry J: Involvement of p38 in apoptosis-associated membrane blebbing and nuclear condensation. Mol Biol Cell 12: 1569-1582, 2001.

3. Pandey P, Raingeaud J, Kaneki M, Weichselbaum R, Davis RJ, Kufe D and Kharbanda S: Activation of p38 mitogen-activated protein kinase by c-Abl-dependent and -independent mechanisms. J Biol Chem 271: 23775-23779, 1996.

4. Winograd-Katz SE and Levitzki A: Cisplatin induces PKB/Akt activation and p38(MAPK) phosphorylation of the EGF receptor. Oncogene 25: 7381-7390, 2006.

5. Franke TF, Hornik CP, Segev L, Shostak GA and Sugimoto C: PI3K/Akt and apoptosis: Size matters. Oncogene 22: 8983-8998, 2003.

6. Kandel ES and Hay N: The regulation and activities of the multifunctional serine/threonine kinase Akt/PKB. Exp Cell Res 253: 210-229, 1999.

7. Wang M, Liu ZM, Li XC, Yao YT and Yin ZX: Activation of ERK1/2 and Akt is associated with cisplatin resistance in human lung cancer cells. J Chemother 25: 162-169, 2013.

8. Chi C, He ZF, Liu Y, Lin XM and Sun CC: Expression and clinical significance of circadian gene Per2 in non-small cell lung cancer. Zhonghua Zhong Liu Za Zhi 35: 129-131, 2013 (In Chinese).

9. Liu B, Xu K, Jiang Y and Li X: Aberrant expression of Per1, Per2 and Per3 and their prognostic relevance in non-small cell lung cancer. Int J Clin Exp Pathol 7: 7863-7871, 2014.

10. Okabe T, Kumagai M, Nakajima Y, Shirotake S, Kodaira K, Oyama M, Ueno M and Ikeda M: The impact of HIF1 $\alpha$ on the Per2 circadian rhythm in renal cancer cell lines. PLoS One 9: e109693, 2014

11. Zhao H, Zeng ZL, Yang J, Jin Y, Qiu MZ, Hu XY, Han J, Liu KY, Liao JW, Xu RH and Zou QF: Prognostic relevance of Period1 (Per1) and Period2 (Per2) expression in human gastric cancer. Int J Clin Exp Pathol 7: 619-630, 2014.

12. Esposito L, Conti D, Ailavajhala R, Khalil N and Giordano A: Lung Cancer: Are we up to the Challenge? Curr Genomics 11: 513-518, 2010.

13. Fu L and Lee CC: The circadian clock: Pacemaker and tumour suppressor. Nat Rev Cancer 3: 350-361, 2003.

14. Yang X, He X, Yang Z and Jabbari E: Mammalian PER2 regulates AKT activation and DNA damage response. Biochem Cell Biol 90: 675-682, 2012.

15. Chen ST, Choo KB, Hou MF, Yeh KT, Kuo SJ and Chang JG: Deregulated expression of the PER1, PER 2 and PER 3 genes in breast cancers. Carcinogenesis 26: 1241-1246, 2005.

16. Sjöblom T, Jones S, Wood LD, Parsons DW, Lin J, Barber TD, Mandelker D, Leary RJ, Ptak J, Silliman N, et al: The consensus coding sequences of human breast and colorectal cancers. Science 314: 268-274, 2006. 